\title{
Estrategias de pensamiento constructivo en estudiantes universitarios"
}

\author{
Strategies of Constructive Thinking in University Students
}

Recibido: mayo 29 de 2009 | Revisado: julio 27 de 2009 ～Aceptado: febrero 4 de 2010

\author{
OfELIA CONTRERAS GUTIÉRREZ ** \\ MARgarita Chávez BECERRA \\ LAURA EDNA ARAgÓN BORJA \\ Miriam VelázQuez OrTIZ \\ Universidad Nacional Autónoma de México
}

Para citar este artículo. Contreras, O., Chávez, M., Aragón, L. E., \& Velázquez, M. (2011). Estrategias de pensamiento constructivo en estudiantes universitarios. Universitas Psychologica, 10(1), 99-111.

Artículo de investigación. Este trabajo es producto del Proyecto de Investigación: "Determinación de la validez predictiva y de constructo de la Escala de Pensamiento Constructivo para Estudiantes Universitarios", financiado por PAPIIT UNAM No. IN404008.

** Facultad de Estudios Superiores, Iztacala. Av. De los Barrios No. 1, Los Reyes Iztacala, Tlalnepantla, Edo. de México, C. P. 54090. E-mails: ofeliaco@gmail. com; margaritachabe@gmail.com; aragonb@gmail. com; mvelazquezortiz@gmail.com

\section{RES UMEN}

El pensamiento constructivo se refiere a la habilidad de generar pensamientos que favorecen la solución de problemas de la vida diaria de manera eficaz, con el menor costo de estrés y un mayor nivel de satisfacción personal. El objetivo de este trabajo fue identificar las estrategias de pensamiento constructivo que utilizan los estudiantes universitarios. En cuanto a pensamiento constructivo, no se encontraron diferencias significativas, pero sí en el modo de afrontar los problemas: los hombres se encaminan a aminorar los efectos emocionales que aquéllos les causan, con más alta autoestima y capacidad de sobreponerse, mientras que las mujeres se encuentran más interesadas en emprender acciones para resolverlos. Por otra parte, los hombres presentan, en comparación con ellas, más alto pensamiento no constructivo, siendo intolerantes, inflexibles y presentando un optimismo ingenuo.

\section{Palabras clave autores}

Pensamiento constructivo, pensamiento no constructivo, estudiantes universitarios, afrontamiento emocional, afrontamiento conductual.

Palabras clave descriptores

Pensamiento, estudiantes universitarios, constructivismo (Psicología).

\footnotetext{
A B S T R A C T

Constructive thinking refers to a person's ability to think in a manner that solves everyday problems with the lowest cost of stress and a higher level of personal satisfaction. The aim of this paper was to identify the strategies of constructive thinking that are used by university students. Results show that there are no significant differences in global constructive thinking between male and female students. Although, exist differences in the copy style: men are better at emotional copies, they diminish the emotional cost of the events, and overcame faster negative experiences, they also show higher self-esteem. Women, on the other hand, show better strategies in order to solve problems (behavioral copy).

Key words authors

Constructive Thinking, Non-constructive Thinking, University Students, Emotional Copy, Behavioral Copy.

Key words plus

Thought and Thinking, University Students, Constructivism (Psychology).
} 
En las últimas décadas, se han desarrollado diferentes aproximaciones teóricas acerca de la inteligencia humana en las que, además de enfatizarse el componente intelectual, se reconoce la importancia de los factores emocionales y de personalidad en el comportamiento inteligente (Epstein, 1998; Salovey \& Meyer, 1990; Stenberg, 1997). Una importante contribución en este campo es la Teoría Cognitivo Experiencial propuesta por Epstein (1998), que se enfoca al estudio y evaluación de las creencias básicas, de carácter preconciente, que orientan la percepción que los individuos tienen acerca de los eventos de la vida cotidiana, así como de las emociones que estos eventos despiertan en ellos, condicionándolos y por tanto predisponiéndolos a actuar con ciertos patrones de comportamiento. Esta teoría sugiere que las emociones de la vida diaria están producidas por la interpretación preconciente de los eventos, derivada de una manera específica de percibir la realidad, es decir, de una cognición, ya que cada persona otorga un significado individual y único a los acontecimientos de la vida cotidiana, que implica los hechos pasados, presentes y futuros.

Epstein (1998) nos habla, en su teoría, de dos tipos de inteligencia, la racional, que se puede medir a través del CI y la inteligencia experiencial, que es la inteligencia relacionada con las emociones y comprende a su vez las inteligencias emocional, social y práctica, equiparando a esta inteligencia experiencial con el pensamiento constructivo. Así, para Epstein, el pensamiento constructivo se refiere a la capacidad para relacionarnos con los demás de un modo efectivo y de forma satisfactoria (inteligencia emocional), solucionar problemas interpersonales de una manera adecuada (inteligencia social) y la capacidad para solucionar eficazmente los problemas que surgen en la vida cotidiana (inteligencia práctica). De esta manera, el pensamiento constructivo es un constructo teórico que hace referencia a la capacidad para reconocer las propias emociones y sentimientos, y al proceso de regulación y dirección consciente y voluntaria que de ellos se haga para la solución de problemas, así como para la consecución de las metas que cada individuo se propone, con el menor costo de estrés, mayor nivel de satisfacción personal y que además resultan adaptativas en el medio social.

Mientras para los teóricos de la inteligencia emocional (Goleman, 1999), esta habilidad corresponde a la esfera de las emociones, se construye en etapas tempranas y no puede ser modificada por el aprendizaje, posteriormente al momento en que se están estableciendo las conexiones neuronales correspondientes (las denominadas ventanas de oportunidad), para otros teóricos este constructo corresponde de manera más directa al terreno de la cognición y, por tanto, puede ser modificado a través del aprendizaje y, si es necesario a través de la resignificación que se promueva por medio de procesos terapéuticos. Martínez-Otero (2002) denomina a este constructo como inteligencia afectiva, la define como: "la capacidad para conocer, expresar y controlar la afectividad, sobre todo los sentimientos, las emociones, las pasiones y las motivaciones" (p. 82), y señala que la conforman tres tipos de competencias: cognitiva, afectiva y conductual.

La competencia cognitiva es la capacidad para utilizar el pensamiento de forma eficaz y constructiva. Incluye los procesos mentales de comprensión, razonamiento, abstracción, resolución de problemas, aprendizaje de la experiencia y adaptación al entorno. Especialmente relevante es la metacognición o capacidad para pensar en la propia cognición y controlarla. En el marco de la inteligencia afectiva, la competencia cognitiva se caracteriza por una orientación prosocial, pues el conocimiento y la habilidad que se poseen tienden a reforzar los lazos interpersonales, es decir, la convivencia.

La competencia afectiva es la capacidad para reconocer, expresar y canalizar la vida emocional. Adquiere especial importancia el equilibrio personal, la autoestima y la empatía. También es importante la meta-afectividad o capacidad de la persona para conocer y gobernar los sentimientos que provocan los fenómenos afectivos. Este componente se refiere sobre todo a las habilidades que tiene el sujeto para comprender la afectividad y, en consecuencia, enriquecer la propia vida.

Por último, la competencia conductual se refiere a las acciones que realiza el sujeto, a partir de 
su pensamiento y de su afectividad. La planificación es básica para que no nos hallemos, más allá de lo imprescindible, en meros automatismos. La estructura cognitivo-emocional equilibrada y rica libera de la "robotización" y facilita la aparición de conductas positivas a nivel personal, profesional, social, etc.

Desde las teorías de la inteligencia afectiva, se resalta la percepción, la comprensión y la regulación emocional para la adaptación al entorno y se reconoce que contribuyen sustancialmente al bienestar psicológico y al crecimiento personal; asimismo, se acepta que influyen de manera importante en el uso del potencial intelectual que poseen las personas (CI) así como en el rendimiento académico del alumnado (Mayer \& Salovey, 1997; Salovey \& Mayer, 1990).

Es comúnmente aceptado que las personas con elevada inteligencia afectiva tienen más posibilidades de adaptarse a las situaciones y de obtener éxito en sus proyectos y que, por el contrario, las personas con poca inteligencia emocional tienden a percibir el mundo, a sí mismos y al futuro de una manera negativa, lo que los predispone a actuar de manera poco adaptativa, así como a seleccionar estrategias inadecuadas para la solución de problemas y la construcción de un autoconcepto pobre; incluso en algunos casos, tienden a la depresión y a la desesperanza (Martínez-Otero, 2002).

La inteligencia afectiva, de acuerdo con Epstein (1998), se sostiene en un conjunto de esquemas de la realidad, de carácter preconsciente y automático, que determinan la manera como percibimos los acontecimientos, al mismo tiempo sirven de soporte a la personalidad. De acuerdo con este autor, de la manera específica como percibimos los acontecimientos se deriva una emoción, y ésta a su vez orienta a la persona a actuar en un sentido específico en concordancia con esa visión y la emoción que se asocia.

Epstein (1998) considera que este sistema de procesamiento de la información actúa de manera paralela al sistema racional, y tiene sus raíces en experiencias tempranas, altamente cargadas de emoción; el principio que rige a este sistema es el de la asociación (si un acontecimiento sigue a otro, el primero causa al segundo) y no el de la lógica, tal como sucede con el sistema racional. Es importante señalar que la teoría de Epstein subraya el carácter cognoscitivo de la inteligencia afectiva, por lo que se refiere a ésta con el término de pensamiento constructivo; con fundamento en esta teoría, Epstein desarrolló el Inventario de Pensamiento Constructivo (CTI).

En el sistema de procesamiento experiencial de la realidad, tiene un lugar preponderante la manera en como las personas se perciben a sí mismas (sus capacidades y posibilidades), al mundo (cómo entendemos la problemática social y qué papel asumimos frente a ella) y al futuro (la posibilidad o no de que nuestras acciones puedan orientarnos en el sentido deseado). De este modo, en el pensamiento no constructivo o destructivo, los pensamientos automáticos se refieren al mundo como un lugar peligroso, en el que no se puede confiar en la gente y en el que cualquier esfuerzo será inútil, y este tipo de pensamiento traerá como consecuencia conductas poco adaptativas y alejadas de las metas que las mismas situaciones sociales les presentan, ya sea en el ámbito académico o extra académico, en contraposición con un pensamiento automático contrario o pensamiento constructivo.

Tales patrones automáticos de pensamiento no solo tendrán una profunda influencia en los sentimientos y comportamientos espontáneos, sino que sesgarán los intentos de pensar racional y objetivamente; influirán no solo en la interpretación de los acontecimientos, sino también en los hechos objetivos que se trata de experimentar como resultado del entorno que se crea a sí misma la persona.

El pensamiento constructivo es una variable que interactúa con algunos otros elementos de la personalidad y con el estilo de las relaciones interpersonales que se establecen; varias investigaciones llevadas a cabo con estudiantes universitarios han mostrado la relevancia de este constructo para explicar la estabilidad emocional de los universitarios, su capacidad de adaptación a la vida escolar y su sentido de "bienestar subjetivo". Por ejemplo, López (1996) analizó la relación entre apego y pensamiento constructivo en universitarios, en su estudio evaluó a una muestra de 145 alumnos de 
las carreras de psicología y educación. De acuerdo con sus hallazgos, los estudiantes que obtienen niveles altos de ansiedad en las relaciones de apego, presentan menores niveles de pensamiento constructivo y de bienestar subjetivo, lo cual repercute en la adaptación a la vida escolar.

En este mismo sentido, algunas investigaciones se han orientado a analizar el pensamiento constructivo de estudiantes, por ejemplo Álvarez y Camargo (2007) evaluaron la relación entre algunas variables de corte demográfico y el pensamiento constructivo en una muestra de 70 estudiantes universitarios. De acuerdo con sus resultados la Rigidez fue la única escala del CTI que se correlacionó significativamente con el nivel socioeconómico de los estudiantes: los de menor nivel económico tendieron a pensar más categóricamente que los de nivel económico alto; por otra parte, los estudiantes de mayor edad presentaron niveles más altos de pensamiento constructivo que los estudiantes más jóvenes. Asimismo, estos autores también encontraron un nivel global de pensamiento constructivo mayor, así como puntuaciones más altas de afrontamiento emocional en hombres que en mujeres, lo que concuerda con los hallazgos de Contreras, Aragón y Velázquez (2008), en un estudio en el que se evaluó el pensamiento constructivo a una muestra de 225 estudiantes universitarios de tres Facultades Multidisciplinarias de las áreas de la salud, administración e ingeniería de la Universidad Nacional Autónoma de México (UNAM); en dicho estudio, los estudiantes del sexo masculino obtuvieron puntajes significativamente más altos en afrontamiento emocional que las mujeres, mientras que los estudiantes del sexo femenino obtuvieron puntajes mayores en afrontamiento conductual.

Por otra parte, también parece existir una tendencia a obtener puntuaciones más altas en eficacia y pensamiento esotérico por parte de las mujeres en comparación con los hombres, ellos tienden a ser ligeramente más rígidos, duros e intolerantes que las mujeres, mientras que ellas tienen una ligera tendencia a estar más dispuestas a aceptar ideas extrañas y a tener un mejor afrontamiento conductual (Álvarez \& Camargo, 2007; Epstein, 1998). Asimismo, Harris y Lightsey (2005) encon- traron que el pensamiento constructivo es un importante predictor de la naturaleza de los afectos, la extroversión y la aparición de neuroticismo en estudiantes. Los buenos pensadores constructivos cuentan con un alto nivel de afecto positivo y felicidad (o satisfacción) y bajos niveles de afecto negativo, lo que concuerda con el estudio llevado a cabo por Scheuer y Epstein (1997), quienes compararon grupos de buenos y malos pensadores constructivos con el CTI, encontrando que las respuestas cognitivas y afectivas de estrés de los participantes con poco pensamiento constructivo fueron más negativas que aquellos de mejor pensamiento constructivo, lo cual apoya la idea de que existe una relación entre el pensamiento constructivo y el bienestar subjetivo.

De acuerdo con estos trabajos, el pensamiento constructivo es un importante mediador entre la personalidad de los estudiantes y el sentido de bienestar subjetivo, que orienta la elección de determinadas estrategias de afrontamiento sobre otras, y con ello promueve distintos niveles de éxito en la resolución de los problemas que la vida académica presenta, con su correspondientes niveles de estrés asociados.

En resumen, cómo vemos al mundo y cómo nos vemos a nosotros mismos en él determina la posibilidad de actuar o no de manera exitosa en la resolución de problemas en la vida diaria. El pensamiento es constructivo cuando nos ayuda a lograr nuestras metas y evitar la perturbación emocional, y es destructivo en el caso contrario. Por ejemplo: "Pienso que no se puede confiar en nadie" o "Pienso que en el fondo todo el mundo es bueno", representan orientaciones generales opuestas que dirigirán nuestro comportamiento hacia los demás en sentidos contrarios. Si alguien piensa que no controla su vida, que es una marioneta del destino y que éste no le depara nada bueno, hay muchas probabilidades de que frustre sus propias metas y su felicidad y que acabe teniendo algún tipo de perturbación emocional, como la depresión. Quien piensa que nada malo puede pasarle y que los problemas al final se arreglan solos sin hacer nada, es muy probable que acabe teniendo dificultades al ver las cosas de un modo tan poco realista, y que 
su conducta, por tanto, sea poco adaptativa. Este conjunto de esquemas que se constituyen en un dispositivo que filtra la información por medio de un sistema atribucional, tiene vital importancia para el desempeño académico y para la satisfacción en la vida dentro de la escuela.

En el ámbito académico los alumnos que tienen muy bajo pensamiento constructivo, no logran desarrollar todo el potencial intelectual indicado por su CI; parecería que su incapacidad de pensar constructivamente interfiriera con el uso de su capacidad intelectual. Es evidente que un alumno que no logra relacionarse adecuadamente con sus maestros y sus compañeros, que exhibe una confianza excesiva o sostiene una actitud derrotista, $\mathrm{o}$ que se agota psíquica e incluso físicamente por el estrés, correrá con desventajas en sus estudios. Por el contrario, en los estudios realizados por Epstein (1998), se ha visto que un buen pensamiento constructivo está relacionado con el éxito en el trabajo, en la salud física, el ajuste emocional, el éxito en las relaciones personales y una mayor satisfacción en la vida en general.

Las autoras del presente artículo suponen que este conjunto de esquemas que poseen todas las personas sobre sí mismas, sobre el mundo y sobre sus expectativas del futuro, constituyen un sistema atribucional, que se asienta en el constructo teórico pensamiento constructivo, al cual visualizan como un continuo que va desde el extremo negativo hasta el extremo positivo, y que para cada persona toma valores específicos, en función de su construcción personal. Con base en estos hallazgos y con la finalidad de contar con un instrumento ad hoc para evaluar el pensamiento constructivo en estudiantes universitarios de manera confiable y válida, se construyó el Cuestionario de Pensamiento Constructivo para Estudiantes Universitarios (CPCE), enmarcado en la definición de pensamiento constructivo de Epstein, así como en la estructura del Cuestionario de Pensamiento Constructivo (CTI) y orientado a eventos o situaciones vinculados específicamente a la vida académica, y cuyo objetivo es evaluar el grado en el cual los pensamientos automáticos preconscientes de los estudiantes favorecen o no la solución de problemas que la vida académica les presenta (Contreras, Chávez \& Velázquez, 2006). De esta manera, el objetivo de este trabajo es identificar las estrategias de pensamiento constructivo que utilizan los estudiantes universitarios, medido a través del CPCE.

\section{Método}

\section{Población}

Estudiantes de nivel licenciatura de los cinco Campus Multidisciplinarios de la UNAM, ubicados en la zona metropolitana de la ciudad de México.

\section{Selección de la muestra}

Se llevó a cabo un muestreo no probabilístico por cuotas. El tamaño de la muestra se estableció en un mínimo del $10 \%$ de la población total en cada una de las carreras; en los casos en que la carrera tuviera menos de 1.000 alumnos, la cuota se estableció en 100 alumnos.

\section{Instrumento}

Cuestionario de Pensamiento Constructivo para Estudiantes Universitarios (CPCE), el cual cuenta con un total de 73 ítems en un formato tipo Likert con cinco opciones de respuesta, que van del total desacuerdo al total acuerdo.

Como ya mencionamos, el CPCE está sustentado teóricamente en la Teoría CognitivoExperencial de Epstein (1998) y su construcción se realizó con base en la estructura del Inventario de Pensamiento Constructivo (CTI) propuesto por el mismo autor; sin embargo, los contenidos del CPCE, a diferencia del CTI, se dirigen específicamente a situaciones de la vida escolar y su finalidad es identificar el tipo de pensamientos de carácter constructivo o no constructivo que intervienen en la solución de problemas que la vida académica presenta a los estudiantes.

Los contenidos de escalas y subescalas iniciales del CPCE se obtuvieron de autorreportes de un grupo de estudiantes acerca de los pensamientos 
TABLA 1

Contribución de cada licenciatura al tamaño de la muestra

\begin{tabular}{|c|c|c|c|c|c|c|c|c|c|}
\hline \multicolumn{10}{|c|}{ Campus Multidisciplinario } \\
\hline \multicolumn{2}{|l|}{ Acatlán } & \multicolumn{2}{|l|}{ Aragón } & \multicolumn{2}{|l|}{ Cuautitlán } & \multicolumn{2}{|l|}{ Iztacala } & \multicolumn{2}{|l|}{ Zaragoza } \\
\hline Licenciatura & $\mathrm{n}$ & Licenciatura & $\mathrm{n}$ & Licenciatura & $\mathrm{n}$ & Licenciatura & $\mathrm{n}$ & Licenciatura & $\mathrm{n}$ \\
\hline \multirow{2}{*}{ Arquitectura } & \multirow{2}{*}{66} & \multirow{2}{*}{$\begin{array}{l}\text { Ingeniería } \\
\text { Mecánica }\end{array}$} & \multirow{2}{*}{82} & Administración & 115 & Biología & 120 & \multirow{2}{*}{$\begin{array}{l}\text { Cirujano } \\
\text { Dentista }\end{array}$} & \multirow{2}{*}{86} \\
\hline & & & & Contaduría & 86 & Medicina & 85 & & \\
\hline \multirow{2}{*}{$\begin{array}{l}\text { Derecho } \\
\text { Diseño Grafico }\end{array}$} & 95 & Pedagogía & 92 & Ingeniería en & \multirow{2}{*}{72} & & & Enfermería & 87 \\
\hline & 67 & \multirow{2}{*}{$\begin{array}{l}\text { Relaciones } \\
\text { Internacionales }\end{array}$} & \multirow[b]{2}{*}{37} & & & Optometría & 83 & \multirow{2}{*}{ Psicología } & \multirow{2}{*}{95} \\
\hline $\begin{array}{l}\text { Matemáticas } \\
\text { Aplicadas }\end{array}$ & 80 & & & $\begin{array}{l}\text { Ingeniería } \\
\text { Química }\end{array}$ & 40 & & & & \\
\hline Total & 308 & Total & 211 & Total & 313 & Total & 288 & Total & 268 \\
\hline
\end{tabular}

Fuente: elaboración propia.

automáticos que acompañan a las emociones referidas por ellos y a los pensamientos que ocurren al dar solución a situaciones, tareas y demandas en general de la vida diaria dentro de la escuela (elaboración de trabajos, exposiciones, evaluaciones, interacciones con compañeros y profesores). Las escalas, subescalas y el número de ítems inicial se modificó utilizando procedimientos de análisis factorial y de confiabilidad en estudios previos, para quedar estructurado como se presenta actualmente (Contreras et al., 2006).

El CPCE está formado por seis escalas, dos escalas dirigidas a evaluar pensamientos de tipo constructivo, tres orientadas a la identificación de pensamientos no constructivos y una escala independiente integrada por elementos de todas las escalas anteriores. Las escalas asociadas a la identificación de Pensamiento Constructivo (PC) son Afrontamiento Conductual (ACON) y Afrontamiento Emocional (AEMO), la que a su vez contiene las subescalas Autoestima (Aut) y Capacidad de Sobreponerse (Sob). Las escalas estructuradas para la identificación de Pensamiento No Constructivo (PNC) son Rigidez (RIG), Pensamiento Esotérico (ESO) e Ilusión (ILU); por último, se encuentra la escala independiente (PCG), denominada Pensamiento Constructivo Global (Tabla 2).
La confiabilidad del CPCE obtenida mediante los índices alpha de Cronbach y de dos mitades, muestra índices aceptables tanto para escalas como para subescalas (Tabla 3); en cuanto a los índices Alpha de Cronbach para el total de los ítems orientados a la identificación de Pensamiento Constructivos (PC) y para el total de los orientados a Pensamiento No Constructivo (PNC), ambos son satisfactorios, obteniéndose valores de $\alpha=.78$ y $\alpha=.73$, respectivamente.

El CPCE cuenta con validez de contenido y de estructura interna o validez factorial (Contreras et al., 2006). Actualmente está en proceso el acopio de evidencias de su relación con otros constructos y variables (inteligencia, rendimiento escolar, afrontamiento, adaptación, ansiedad y depresión).

\section{Procedimiento}

Se aplicó el CPCE a la muestra seleccionada, de manera colectiva en los salones de clase, previa autorización de los profesores de cada grupo; no se estableció límite de tiempo para responder al cuestionario, aunque en términos generales la tarea se realizó en 30 minutos. La aplicación del CPCE se llevó a cabo durante el segundo semestre del período escolar del año 2007. 
TABLA 2

Escalas, subescalas, número de ítems y puntuaciones mínimas y máximas

\begin{tabular}{|c|c|c|c|c|c|}
\hline Pensamiento & Escalas & Subescalas & $\begin{array}{l}\text { Número de } \\
\text { ítems }\end{array}$ & $\begin{array}{l}\text { Puntuación } \\
\text { mínima }\end{array}$ & $\begin{array}{l}\text { Puntuación } \\
\text { máxima }\end{array}$ \\
\hline \multirow{3}{*}{$\begin{array}{l}\text { Constructivo } \\
\text { (PC) }\end{array}$} & \multirow{2}{*}{$\begin{array}{l}\text { Afrontamiento emocional } \\
\text { (AEMO) }\end{array}$} & $\begin{array}{l}\text { Autoestima } \\
\text { (Aut) }\end{array}$ & 7 & 7 & 35 \\
\hline & & $\begin{array}{l}\text { Capacidad de sobreponerse } \\
\text { (Sob) }\end{array}$ & 8 & 8 & 40 \\
\hline & $\begin{array}{l}\text { Afrontamietno conductual } \\
(\mathrm{ACON})\end{array}$ & & 12 & 12 & 60 \\
\hline \multirow{3}{*}{$\begin{array}{l}\text { No Constructivo } \\
\text { (PNC) }\end{array}$} & $\begin{array}{l}\text { Rigidez } \\
\text { (RIG) }\end{array}$ & & 7 & 7 & 35 \\
\hline & $\begin{array}{l}\text { Pensamiento esotérico } \\
\text { (ESO) }\end{array}$ & & 8 & 8 & 40 \\
\hline & Ilusión ( ILU) & & 12 & 12 & 60 \\
\hline $\begin{array}{l}\text { Escala Indepen- } \\
\text { diente }\end{array}$ & $\begin{array}{l}\text { Pensamiento } \\
\text { Constructivo } \\
\text { Global (PCG) }\end{array}$ & & 19 & 19 & 95 \\
\hline
\end{tabular}

Nota. Las abreviaturas de identificación de escalas y subescalas se presentan en negritas y entre paréntesis.

Fuente: elaboración propia.

TABLA 3

Índices de confiabilidad del CPCE

\begin{tabular}{cccc}
\hline Escalas y subescalas & No. de ítems & Alpha & 2 mitades \\
\hline PCG & 19 & .84 & .77 \\
AEMO & 15 & .77 & .62 \\
Aut & 7 & .77 & .77 \\
Sob & 8 & .63 & .54 \\
ACON & 12 & .74 & .65 \\
RIG & 7 & .55 & .54 \\
ESO & 8 & .62 & .63 \\
ILU & 12 & .53 & .48 \\
\hline
\end{tabular}

Fuente: elaboración propia. 


\section{Resultados}

Una vez aplicados y calificados los cuestionarios, se elaboraron tablas de baremos para el instrumento; asimismo, se realizaron comparaciones para determinar si existían diferencias significativas entre hombres y mujeres, tanto en Pensamiento Constructivo (PC) como en Pensamiento No Constructivo (PNC), así como en sus diferentes escalas y subescalas.

Con respecto a las tablas de baremos, con base en los criterios psicométricos utilizados con las puntuaciones percentiles, se identificaron las puntuaciones que permitirán clasificar, en aplicaciones futuras del instrumento para evaluaciones individuales, el Pensamiento Constructivo en Adecuado, Alto y Bajo y el Pensamiento No Constructivo como Moderado, Bajo y Alto (puntuaciones medias, altas y bajas, respectivamente). Asimismo, también se encuentran determinadas las puntuaciones de corte en estas categorías, para cada una de las escalas y subescalas del CPCE. Se inserta a continuación un fragmento de la tabla para ilustrar lo anterior, considerando que las puntuaciones medias van del percentil 26 al 74; puntuaciones altas del percentil 75 al 99 y puntuaciones bajas del percentil 1 al 25 (Tabla 4).
En cuanto al porcentaje de mujeres y hombres que obtienen puntuaciones adecuadas, observamos que en Pensamiento Constructivo, es mayor el porcentaje de hombres que de mujeres en puntuaciones adecuadas, pero es mayor el porcentaje de mujeres que de hombres en puntuaciones altas (Tabla 5). Con respecto a Pensamiento No Constructivo, igualmente los hombres presentan un mayor porcentaje de puntuaciones moderadas, pero las mujeres presentan un mayor porcentaje en puntuaciones bajas (Tabla 6).

\section{TABLA 5}

Porcentaje de hombres y mujeres con puntuaciones altas, medias y bajas en Pensamiento Constructivo

\begin{tabular}{lcc}
\hline \multicolumn{1}{c}{ Percentiles } & Hombres & Mujeres \\
\hline $\begin{array}{l}75-99 \\
\text { (Puntuaciones altas) }\end{array}$ & $25.5 \%$ & $26.1 \%$ \\
$\begin{array}{l}26-74 \\
\text { (Puntuaciones medias) }\end{array}$ & $47.9 \%$ & $43.9 \%$ \\
$\begin{array}{l}1-25 \\
\text { (Puntuaciones bajas) }\end{array}$ & $26.6 \%$ & $30 \%$ \\
& $\mathrm{~N}=505$ & $\mathrm{~N}=863$ \\
\hline
\end{tabular}

Fuente: elaboración propia.

TABLA 4

Percentiles y puntuaciones directas para escalas, subescalas y áreas del CPCE

\begin{tabular}{ccccccccccc}
\hline Pc & PCG & PC & AEMO & Aut & Sob & ACON & PNC & RIG & ESO & ILU \\
\hline 5 & 60 & 78 & 39 & 15 & 20 & 33 & 44 & 9 & 9 & 20 \\
10 & 64 & 82 & 43 & 18 & 22 & 35 & 48 & 11 & 10 & 22 \\
12 & 66 & 83 & 44 & 19 & 23 & 36 & 49 & $\ldots$ & 11 & 23 \\
56 & 79 & 99 & 55 & $\ldots$ & $\ldots$ & $\ldots$ & 62 & $\ldots$ & $\ldots$ & 30 \\
59 & 80 & 100 & 56 & $\ldots$ & 30 & 46 & 63 & 17 & $\ldots$ & 30 \\
66 & 82 & 103 & $\ldots$ & 28 & $\ldots$ & 47 & 65 & $\ldots$ & 1 & $\ldots$ \\
96 & 92 & 118 & 68 & $\ldots$ & $\ldots$ & $\ldots$ & 80 & 24 & 24 & 39 \\
97 & 93 & 120 & 69 & 34 & 37 & 55 & 82 & 25 & 25 & $\ldots$ \\
98 & $\ldots$ & 121 & 70 & $\ldots$ & 38 & 56 & 83 & & 26 & 41 \\
\hline
\end{tabular}

Fuente: elaboración propia. 
TABLA 6

Porcentaje de hombres y mujeres con puntuaciones altas, medias y bajas en Pensamiento No Constructivo

\begin{tabular}{lcc}
\hline \multicolumn{1}{c}{ Percentiles } & Hombres & Mujeres \\
\hline $\begin{array}{l}75-99 \\
\text { (Puntuaciones altas) }\end{array}$ & $27.9 \%$ & $21.9 \%$ \\
$\begin{array}{l}26-74 \\
\text { (Puntuaciones medias) }\end{array}$ & $52.1 \%$ & $47 \%$ \\
$\begin{array}{l}1-25 \\
\text { (Puntuaciones bajas) }\end{array}$ & $20 \%$ & $31.1 \%$ \\
& $\mathrm{~N}=505$ & $\mathrm{~N}=863$ \\
\hline
\end{tabular}

Fuente: elaboración propia.

La Tabla 2 nos muestra que las dos grandes áreas que integran el CPCE, PCy PNC, tienen ambas 27 reactivos, por lo que las puntuaciones mínimas y máximas posibles son, respectivamente, 27 y 135. En la distribución generada por las puntuaciones directas de toda la muestra en Pensamiento Constructivo (Figura 1) y en Pensamiento No Constructivo (Figura 2), observamos que, en Pensamiento
Constructivo, no se identificaron alumnos que obtuvieran puntuaciones muy bajas, como las teóricamente posibles, del rango de 27 al 39 (la mínima fue 40) y, en Pensamiento No Constructivo, no se encontraron puntuaciones tan altas del rango de 102 a 135 (la máxima fue 101), lo que significa que los estudiantes en general no presentan conductas relacionadas con un pensamiento constructivo pobre en extremo y, a su vez, no presentan muchas conductas con un alto pensamiento de tipo rígido, esotérico u optimismo ingenuo.

Por último, para las comparaciones entre hombres y mujeres en las diferentes escalas del instrumento, se llevó a cabo una $t$ de Student por medio del programa SPSS v15. La Tabla 7 nos muestra que en PC no existieron diferencias significativas entre hombres y mujeres, ni tampoco en la escala independiente PCG; sin embargo, en Afrontamiento emocional y sus dos subescalas, Autoestima y Capacidad de sobreponerse, sí las hubieron a favor de los hombres, y en Afrontamiento Conductual a favor de las mujeres $(\alpha=.01)$.

\section{FIGURA 1}

Distribución del total de puntuaciones directas. Pensamiento Constructivo

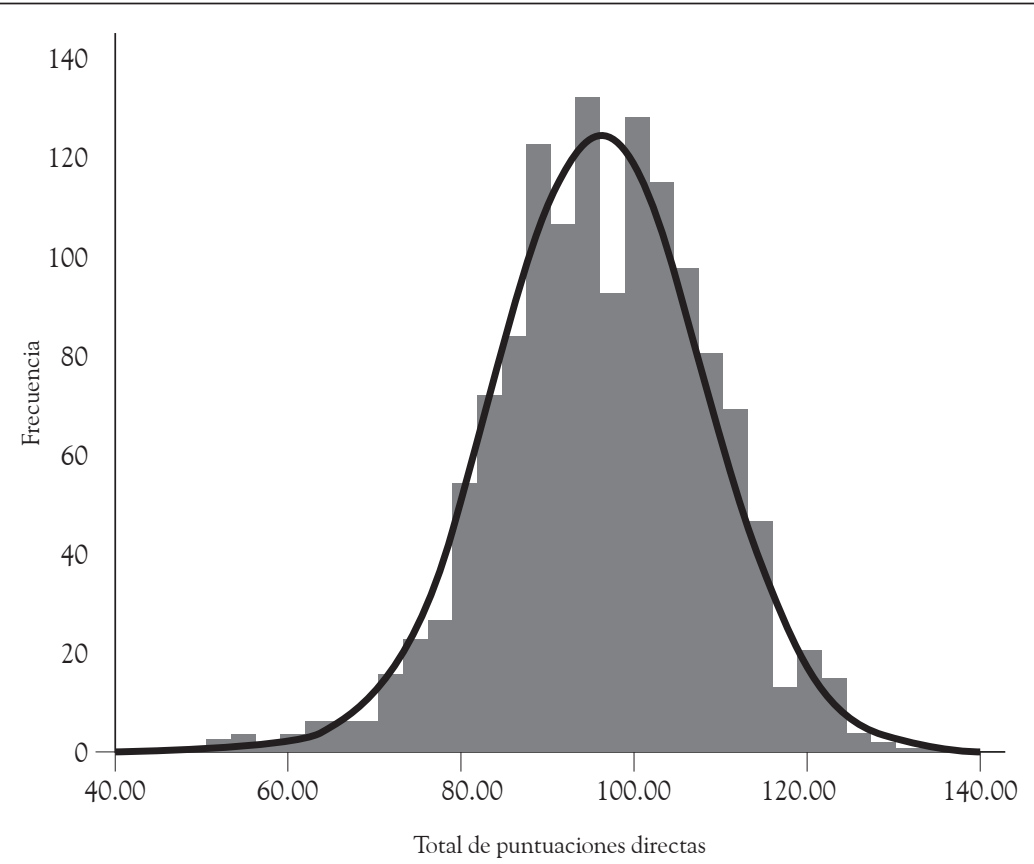

Fuente: elaboración propia. 


\section{TABLA 7}

Comparación de medias de puntuaciones directas de PCG y categorías de Pensamiento Constructivo entre hombres y mujeres

\begin{tabular}{|c|c|c|c|c|c|}
\hline Categorías & Sexo & $\mathrm{N}$ & Media & $T$ & $P$ \\
\hline \multirow{2}{*}{ PCG } & Hombre & 505 & 76.85 & \multirow{2}{*}{-0.55} & \multirow{2}{*}{0.58} \\
\hline & Mujer & 863 & 77.14 & & \\
\hline \multirow{2}{*}{ PC } & Hombre & 505 & 98.00 & \multirow{2}{*}{1.26} & \multirow{2}{*}{0.21} \\
\hline & Mujer & 863 & 97.07 & & \\
\hline \multirow{2}{*}{ AEMO } & Hombre & 505 & 54.98 & \multirow{2}{*}{5.15} & \multirow{2}{*}{$0.00 * *$} \\
\hline & Mujer & 863 & 52.66 & & \\
\hline \multirow{2}{*}{ Aut } & Hombre & 505 & 25.80 & \multirow{2}{*}{3.90} & \multirow{2}{*}{$0.00 * *$} \\
\hline & Mujer & 863 & 4.69 & & \\
\hline \multirow{2}{*}{ Sob } & Hombre & 505 & 29.19 & \multirow{2}{*}{4.66} & \multirow{2}{*}{$0.00 * *$} \\
\hline & Mujer & 863 & 27.97 & & \\
\hline \multirow{2}{*}{$\mathrm{ACON}$} & Hombre & 505 & 42.93 & \multirow{2}{*}{-4.12} & \multirow{2}{*}{$0.00 * *$} \\
\hline & Mujer & 863 & 44.40 & & \\
\hline
\end{tabular}

Fuente: elaboración propia.

FiguRA 2

Distribución del total de puntuaciones directas. Pensamiento No Constructivo

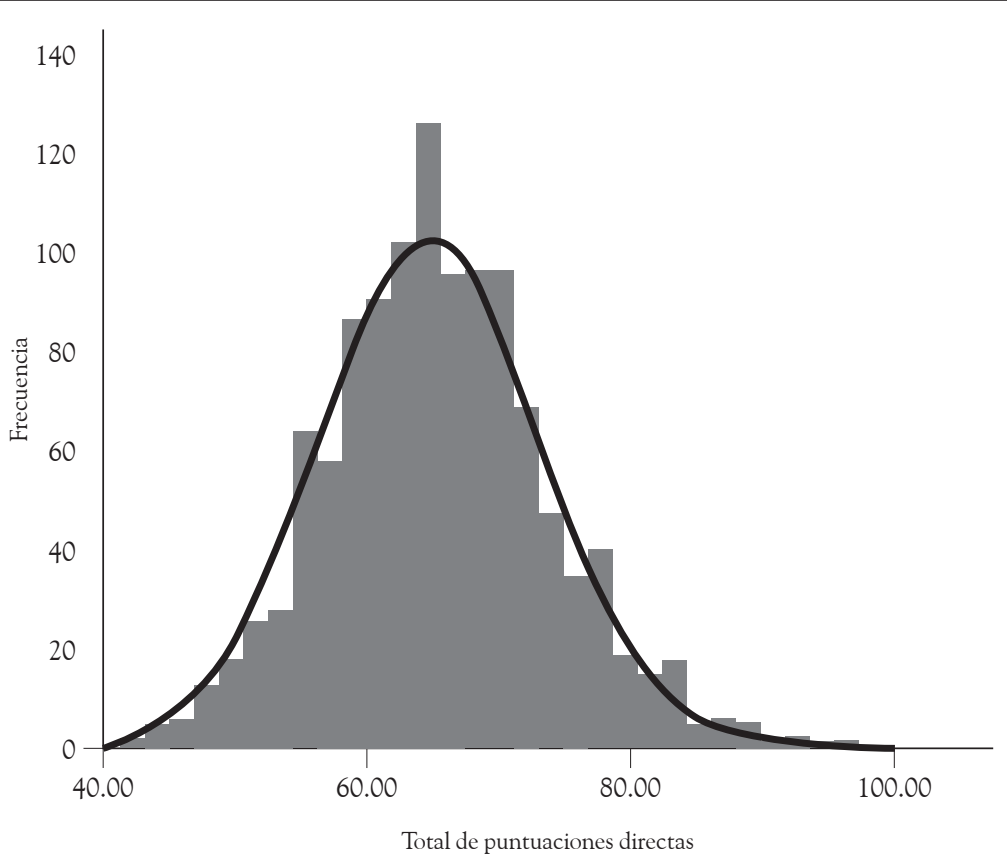

Fuente: elaboración propia. 
Por otra parte, en PNC sí existieron diferencias significativas, presentando los hombres un pensamiento no constructivo más alto en comparación con las mujeres ( $\alpha=.01)$; asimismo, en dos de sus escalas, Rigidez e Ilusión, también presentaron los hombres puntuaciones significativamente más altas que las mujeres ( $\alpha=.01$ y $\alpha=.05$, respectivamente) (Tabla 8$)$.

\section{Discusión}

Si bien no se presentaron puntuaciones extremas inadecuadas en la población estudiada tanto para PC como para PNC, lo que denota que en general los estudiantes universitarios no poseen un pensamiento constructivo demasiado pobre o bien un alto pensamiento destructivo, sin embargo sí existieron algunas diferencias entre hombres y mujeres que hay que destacar.

En cuanto a Pensamiento Constructivo, no se encontraron diferencias significativas entre hombres y mujeres, es decir, ambos poseen en el mismo grado la capacidad de reconocer sus propias emociones, autorregularse y dirigir sus acciones hacia la solución de sus problemas y obtención de sus metas; sin embargo, en cuanto a Pensamiento No
Constructivo sí existen diferencias significativas, presentando los hombres un pensamiento no constructivo más alto en comparación con las mujeres, esto es, perciben en general al mundo, a sí mismos y al futuro, de una manera más negativa que ellas, seleccionando por tanto estrategias menos adecuadas para la solución de sus problemas.

A pesar de que no se encontraron diferencias entre hombres y mujeres en cuanto a Pensamiento Constructivo, sí se encontraron diferencias en sus dos escalas y subescalas. En Afrontamiento Emocional, los hombres presentaron significativamente puntuaciones más altas que las mujeres, lo cual concuerda con los hallazgos de Álvarez y Camargo (2007), así como con los de Contreras, Aragón y Velázquez (2008). Esto es, los hombres, para resolver los problemas que se les presentan, utilizan estrategias que, más que ir encaminadas a la solución de los conflictos, van encaminadas a aminorar los efectos emocionales del problema y a reducir el estrés; el afrontamiento emocional se relaciona con la capacidad de manejar adecuadamente los sentimientos negativos, lo que los hace no ser demasiado sensibles al rechazo ni al fracaso, y no preocuparse en exceso por cosas que no pueden controlar.

\section{TABLA 8}

Comparación de medias de puntuaciones directas de categorías de Pensamiento No Constructivo entre hombres y mujeres

\begin{tabular}{|c|c|c|c|c|c|}
\hline Categorías & Sexo & $\mathrm{N}$ & Media & $T$ & $P$ \\
\hline \multirow{2}{*}{ PNC } & Hombre & 505 & 62.28 & \multirow{2}{*}{3.98} & \multirow{2}{*}{$0.00 * *$} \\
\hline & Mujer & 863 & 59.96 & & \\
\hline \multirow{2}{*}{ RIG } & Hombre & 505 & 16.64 & \multirow{2}{*}{5.13} & \multirow{2}{*}{$0.00 * *$} \\
\hline & Mujer & 863 & 15.43 & & \\
\hline \multirow{2}{*}{ ESO } & Hombre & 505 & 15.93 & \multirow{2}{*}{1.65} & \multirow{2}{*}{0.100} \\
\hline & Mujer & 863 & 15.53 & & \\
\hline \multirow{2}{*}{ ILU } & Hombre & 505 & 29.70 & \multirow{2}{*}{2.37} & \multirow{2}{*}{$0.018^{*}$} \\
\hline & Mujer & 863 & 28.99 & & \\
\hline $\begin{array}{l}* p<0.05 \\
* * p<0.01\end{array}$ & & & & & \\
\hline
\end{tabular}


Por otro lado, en Afrontamiento Conductual, esta relación se invierte, teniendo aquí las mujeres puntuaciones significativamente más altas que los hombres, al igual que en el estudio de Contreras et al. (2008), lo cual nos muestra que las mujeres, en comparación con los hombres, más que interesadas en aminorar los efectos emocionales de los problemas que se les presentan, intentan realizar acciones encaminadas a resolverlos; es un pensamiento orientado a la acción, con la finalidad de encontrar formas efectivas de solucionar problemas, aceptando retos y arriesgándose.

Con respecto a las dos subescalas de Afrontamiento Emocional, en congruencia con lo anterior y como era de esperarse, los hombres presentaron puntuaciones significativamente más altas que las mujeres, tanto en Autoestima como en Capacidad de sobreponerse, esto es, los hombres son más seguros de sí mismos, se consideran más valiosos y poseen un mejor autoconcepto que las mujeres y no les afecta tanto como a ellas la aprobación o desaprobación de los demás; asimismo, se angustian o preocupan en menor grado que sus compañeras por problemas de difícil solución o por las equivocaciones cometidas, pensando que a futuro tendrán la capacidad de resolverlos.

Por último, en dos de las escalas de Pensamiento No Constructivo, Rigidez e Ilusión, los hombres presentaron puntuaciones significativamente más altas que las mujeres; esto era de esperarse, ya que los hombres presentaron puntuaciones más altas en Pensamiento No Constructivo que ellas. Así, podemos decir que en esta investigación, los hombres, en comparación con las mujeres, tienen un tipo de pensamiento más rígido, que se caracteriza por no ser flexible en cuanto a los juicios que hacen de los demás, siendo intolerantes, no admitiendo sino su punto de vista y catalogando a las opiniones diferentes a las suyas como errores, su pensamiento es absolutista y dicotómico (blanco o negro, bueno o malo, perdedores o ganadores, amigos o enemigos); con respecto a Ilusión, en comparación con las mujeres, los hombres poseen un tipo de optimismo más ingenuo, sin fundamento, creyendo que para obtener cosas en la vida basta con desearlo y con tener buena suerte, teniendo una visión simplista de la realidad, por lo que evitan las realidades desagradables y no toman las debidas precauciones.

Las escalas donde no se observaron diferencias significativas fueron Pensamiento Constructivo Global y Pensamiento esotérico; lo primero es congruente con no haber encontrado diferencias en Pensamiento Constructivo; y lo segundo, significa que tanto los hombres como las mujeres poseen en el mismo grado un pensamiento de tipo esotérico (fenómenos paranormales o extrasensoriales) y supersticioso relacionado con la solución de problemas.

\section{Conclusiones}

En Pensamiento Constructivo, no existieron diferencias significativas entre hombres y mujeres, aunque sí en el modo de afrontar los problemas: los hombres se encaminan a aminorar los efectos emocionales y el estrés que aquéllos les causan (afrontamiento emocional), buscando apoyo emocional y teniendo mejor autoestima y capacidad de sobreponerse, mientras que las mujeres se encuentran más interesadas en emprender acciones para resolverlos (afrontamiento conductual).

Sin embargo, en Pensamiento No Constructivo, los hombres sí presentan significativamente más pensamientos de este tipo en comparación con las mujeres, obteniendo asimismo puntuaciones significativamente más altas en las Escalas de Rigidez e Ilusión, lo que los hace ser más intolerantes e inflexibles con los demás, así como poseer un optimismo más ingenuo que las mujeres.

De los resultados encontrados concluimos que tanto los hombres como las mujeres estudiantes universitarios poseen en el mismo grado pensamientos de tipo constructivo, aunque siendo los hombres mejores afrontadores emocionales, con más alta autoestima y capacidad de sobreponerse y las mujeres mejores afrontadoras conductuales; habría que enseñarles a cada uno a mejorar en el tipo de habilidades de afrontamiento que les hace falta, ya que las autoras del presente estudio consideran que, para resolver con éxito los problemas que se presentan en la vida académica, ambos tipos 
de afrontamiento, el conductual y el emocional, son indispensables y se complementan.

Por otra parte, los hombres presentan en comparación con ellas más alto pensamiento no constructivo, con pensamientos de tipo rígido y optimismo ingenuo. Aquí, habría que trabajar con ellos para enseñarles a ser más flexibles y menos dogmáticos, así como a enfrentar los acontecimientos de una manera más realista y menos idealista.

\section{Referencias}

Álvarez, L. \& Camargo, J. (2007). Pensamiento constructivo y variables sociodemográficas en estudiantes de primero a tercer semestre de la facultad de psicología de la Universidad Autónoma de Bucaramanga. Psicología desde el Caribe, 19, 203-221.

Contreras, G. O., Aragón, B. L. \& Velásquez, O. M. (2008). El pensamiento constructivo en estudiantes universitarios: una evaluación con el CPCE. Ponencia presentada en el XV Congreso Internacional de Investigación Educativa, Tampico, Tamaulipas, México.

Contreras, G. O., Chávez, B. M. \& Velásquez, O. M. (2006). Cualidades psicométricas del Cuestionario de Pensamiento Constructivo para Estudiantes Universitarios. Revista de la Sociedad de ex Alumnos de la Facultad de Psicología, 9(1-2), 13-22.

Epstein, S. (2001). Inventario de Pensamiento Constructivo (CTI). Una medida de la Inteligencia Emocional. [Manual]. Madrid: TEA Ediciones.
Epstein, S. (1998). Constructive thinking. The key of emotional intelligence. London: Paeger.

Goleman, D. (1999). Inteligencia Emocional. Madrid: Kairós.

Harris, P. \& Lightsey, O. (2005). Constructive thinking as a mediator of the relationship between extraversion, neuroticism, and subjective well-being. European Journal of Personality, 19(5), 409-426.

López, G. (1996). Attachment-related predictors of constructive thinking among college students. Journal of Counseling and Development, 75(1), 58-63.

Mayer, J. D. \& Solovey, P. (1997). What is emotional intelligence? En P. Solov \& D. Sluyter (Eds.), Emotional development and emotional intelligence. Implications for educators (pp. 3-31). New York: Basic Books.

Martínez-Otero, V. (2002). Reflexiones psicopedagógicas sobre la inteligencia. Pulso: Revista de Educación, 25, 77-86.

Salovey, P. \& Mayer, J. D. (1990). Emotional intelligence. Imagination, Cognition, and Personality, 9, 185-211.

Scheuer, E. \& Epstein, S. (1997). Constructive thinking, reactions to a laboratory stressor, and symptoms in everyday life. Anxiety, Stress and Coping, 10(3), 269-303.

Stenberg, R. J. (1997). A triarchic view of giftedness: Theory and practice. In N. Coleangelo \& G. A. Davis (Eds.), Handbook of Gifted Education (pp. 43-53). Boston, MA: Allyn and Bacon. 
